\title{
Semiparametric Methods of Time Scale Selection
}

\author{
Thierry Duchesne \\ University of Toronto, Toronto, Canada
}

\begin{abstract}
In several reliability applications, there may not be a unique plausible scale in which to analyze failure. In this paper, I consider semiparametric methods of time scale selection. I propose a rank-based estimator of the time scale parameters that can readily handle censored observations. I illustrate how to assess the form of the time scale through generalized residuals. I also give ideas for nonparametric estimation of the time scale.
\end{abstract}

Keywords and phrases: collapsible model, generalized residuals, ideal time scale, survival analysis, quantile regression, rank regression, reliability, usage path.

\section{$1.1 \quad$ Introduction}

In many reliability applications, there may not be a unique plausible time scale in which to assess performance or model failure. For example, should we measure the age of an automobile in calendar time since purchase or in cumulative mileage, or perhaps even in a function of these two measures [Lawless et al. (1995)]? Should we measure the age of an aircraft in calendar time, cumulative flight time, or number of landings [Kordonsky and Gertsbakh (1993, 1995ab, 1997)]? Should we measure the lifetime of miners exposed to asbestos dust in biological age or in cumulative exposure [Oakes (1995)]?

Even though it is possible to model most processes with respect to chronological time, there are some advantages in adopting an alternative scale $t$ in certain contexts [Farewell and Cox (1979), Duchesne and Lawless (2000)]. To name a couple:

1. the failure mechanism itself may depend primarly on $t$, so that it is in some way "scientifically sufficient". For example, the probability that an 
item fails after some chronological time $x$ may depend only on its age in the alternative scale $t$ at time $x$;

2. the effect of treatments or covariates may be most simply expressed on some scale other than real time.

In this paper, I present some semiparametric methods of inference for a special class of models emphasizing simple time scales introduced by Oakes (1995). In Section 2, the concept of ideal time scale is presented and a class of simple models is considered. I derive semiparametric estimators of the time scale parameters for these models in Section 3. Semiparametric model assessment from generalized residuals is illustrated through an example in Section 4. I provide ideas for completely nonparametric estimation of the ideal time scale in Section 5. Some concluding remarks are given in Section 6 .

\subsection{Modeling failure}

Let $x$ denote chronological time from an origin that usually corresponds to the birth or introduction of a unit into service. Let $X$ represent the random variable of chronological time or age at failure for a unit.

Definition 1.2.1 A usage measure is a time-varying covariate, say $z(x)$, such that

- $z(x)$ is left-continuous;

- $z(x)$ is non-decreasing in $x$;

- $z(0)=0$

- $z(\cdot)$ is external, i.e. its history is determined independently of the failure time $X$.

Examples of usage measures include the cumulative mileage on an automobile, the cumulative number of landings of an aircraft and the cumulative amount of exposure of a rat to a potential carcinogen.

Now suppose that for each unit under study, the usage path (or history) is available, i.e. we are given $\mathcal{Z}=\left\{z_{1}(x), \ldots, z_{p}(x) ; x \geq 0\right\}$ for every unit, where $z_{1}(x), \ldots, z_{p}(x)$ are $p$ usage measures. Let $\mathbf{Z}$ represent the set of all possible usage paths. We conceive of a time scale (TS) as a function of chronological time and usage measures. Formally, we consider a TS to be a non-negative-valued functional $\Phi: \mathbb{R}^{+} \otimes \mathbf{Z} \rightarrow \mathbb{R}^{+}$that maps $(x, \mathcal{Z})$ to $\Phi[x, \mathcal{Z}]$ and such that $\Phi[x, \mathcal{Z}]$ is non-decreasing in $x$ for all $\mathcal{Z} \in \mathbf{Z}$. We use the notation $t_{\mathcal{Z}}(x)=\Phi[x, \mathcal{Z}]$ to 
emphasize that $\Phi[x, \mathcal{Z}]$ is the time (age) in the $\Phi$-scale of a unit on path $\mathcal{Z}$ at chronological time $x$.

Usage paths are assumed to vary from unit to unit in some population. The fact that they are external allows us to investigate the distribution of time to failure conditional on the realized path $\mathcal{Z}$. Some advantages of such a conditional approach is that it is valid for experiments where the usage histories are fixed by study design, and a single model may be used for populations with dissimilar usage accumulation tendencies.

The following definition of an ideal time scale has been considered by several authors $^{1}$ [Cinlar and Ozekici (1987), Kordonsky and Gertsbakh (1993, 1995ab, 1997), Bagdonavičius and Nikulin (1997a) and Duchesne and Lawless (2000)] and it relates an ideal time scale to the conditional probability of survival given the usage history:

Definition 1.2.2 Let $T=\Phi[X, \mathcal{Z}]$ represent the random variable of time to failure measured in scale $\Phi$. Then $\Phi$ defines an ideal time scale (ITS) if for all $\mathcal{Z} \in \mathbf{Z}$ and all $t \geq 0$ such that $t=\Phi[x, \mathcal{Z}]$ for some $x \geq 0$,

$$
\begin{aligned}
\operatorname{Pr}[X>x \mid \mathcal{Z}] & =\operatorname{Pr}[T>t] \\
& =G(t),
\end{aligned}
$$

where $G(\cdot)$ is a strictly decreasing survivor function.

This defines an ITS as any time scale that is a one-to-one function of the conditional survivor function $\operatorname{Pr}[X>x \mid \mathcal{Z}]$. A consequence of Definition 1.2.2 that will be useful for inference later on is that if $\Phi$ is an ideal time scale, then $\operatorname{Pr}[T>t \mid \mathcal{Z}]=\operatorname{Pr}[T>t]$, which means that the failure times in the ITS, though functionally dependent of $\mathcal{Z}$, are statistically independent of the usage paths.

Let us now consider the cases where $\operatorname{Pr}[X>x \mid \mathcal{Z}]$ is continuous and differentiable in $x$ for all $x \geq 0$ and $\mathcal{Z} \in \mathbf{Z}$. Then one approach to modeling is to incorporate the effect of $\mathcal{Z}$ on $X$ through the conditional hazard function:

$$
\operatorname{Pr}[X>x \mid \mathcal{Z}]=\exp \left\{-\int_{0}^{x} h(u \mid \mathcal{Z}(u)) d u\right\}
$$

where $\mathcal{Z}(x)=\left\{z_{1}(u), \ldots, z_{p}(u) ; 0 \leq u<x\right\}$. We can rewrite (1.2) so as to emphasize an ITS:

$$
\operatorname{Pr}[X>x \mid \mathcal{Z}]=G(\Phi[x, \mathcal{Z}]),
$$

where $G$ is a strictly decreasing survivor function and $\Phi[x, \mathcal{Z}]$ is an ITS for $\operatorname{Pr}[X>x \mid \mathcal{Z}]$. The two models described in (1.2) and (1.3) are equivalent and the relationship between the ITS in (1.3) and the conditional hazard function in (1.2) is given by

$$
h(x \mid \mathcal{Z}(x))=h_{G}(\Phi[x, \mathcal{Z}]) \frac{d}{d x} \Phi[x, \mathcal{Z}]
$$

\footnotetext{
${ }^{1}$ These authors do not necessarily use the term ideal time scale, however.
} 
where $h_{G}(u)=-d \ln G(u) / d u$ is the hazard function corresponding to survivor function $G$.

Generally, models with a simple form for $h(x \mid \mathcal{Z}(x))$ will have a complex form for $\Phi[x, \mathcal{Z}]$ and vice-versa. In this paper, we consider a class of models for which $\Phi[x, \mathcal{Z}]$ is of a simple form. This class of model was introduced by Oakes (1995) and is defined as follows:

Definition 1.2.3 The model $\operatorname{Pr}[X>x \mid \mathcal{Z}]$ is collapsible in $x, z_{1}(x), \ldots, z_{p}(x)$ if

$$
\operatorname{Pr}[X>x \mid \mathcal{Z}]=f\left(x, z_{1}(x), \ldots, z_{p}(x)\right), \quad \forall x \geq 0, \mathcal{Z} \in \mathbf{Z},
$$

where $f: \mathbb{R}^{(p+1)+} \rightarrow \mathbb{R}^{+}$is non-decreasing in $x, z_{1}(x), \ldots, z_{p}(x)$.

This definition means that for a collapsible model, the ITS's will be of the form $\Phi\left[x, z_{1}(x), \ldots, z_{p}(x)\right]$, i.e. the probability of surviving past a certain time $x$ depends on the value of the usage measures at $x$ only, not on their past history. This is a rather strong assumption and we will see how it can be verified in Section 4.

\subsection{Semiparametric inference}

Usually, we specify an ITS up to a finite vector of unknown parameters that are to be estimated from observed data. Of special interest is the case where we observe $\left(x_{1}, \mathcal{Z}_{1}, \delta_{1}\right), \ldots,\left(x_{n}, \mathcal{Z}_{n}, \delta_{n}\right)$, where $\delta_{i}=1$ if $x_{i}$ is an observed failure time and $\delta_{i}=0$ if $x_{i}$ is a right-censoring time, $i=1,2, \ldots, n$, and where we postulate the model

$$
\operatorname{Pr}[X>x \mid \mathcal{Z}]=G\left(\Phi\left[x, z_{1}(x), \ldots, z_{p}(x) ; \boldsymbol{\eta}\right]\right)
$$

where $\boldsymbol{\eta}$ is a finite vector of unknown parameters and $G$ is a strictly decreasing survivor function left unspecified. Oakes (1995) considered inference in the case where $G$ was specified up to a finite vector of parameters.

Kordonsky and Gertsbakh $(1993,1995 \mathrm{ab}, 1997)$ propose a method of estimation for $\boldsymbol{\eta}$ when $G$ is left unspecified in (1.5). Let $t_{\mathcal{Z}_{i}}\left(x_{i} ; \boldsymbol{\eta}\right)=\Phi\left[x_{i}, z_{1, i}, \ldots, z_{n, i} ; \boldsymbol{\eta}\right]$, $i=1, \ldots, n$. Then Kordonsky and Gertsbakh propose to use the value of $\boldsymbol{\eta}$ that minimizes the squared sample coefficient of variation of the $t_{\mathcal{Z}_{i}}\left(x_{i} ; \boldsymbol{\eta}\right)$ 's as an estimate of $\boldsymbol{\eta}$. This method is numerically simple, yields consistent estimators in several frameworks, and a time scale with small coefficient of variation may be desirable in some engineering applications. However, Kordonsky and Gertsbakh (1995b, 1997) have to assume a parametric form for $G$ when some observations in the sample are censored. Defining a censored nonparametric version of the coefficient of variation is also a problem, as extrapolation of the 
tail of the distribution is needed when the largest time in the sample is a censoring time. Moreover, it is not clear that this method is efficient, as investigated by Duchesne and Lawless (2000).

As an alternative, we can adapt a rank-based method derived for the accelerated failure time model proposed by Robins and Tsiatis (1992) and Lin and Ying (1995). This method shows good efficiency properties and can readily handle censoring. Furthermore, the estimating functions obtained are derived directly from the conditional probability model given in (1.5) and in cases where they have a simple form, they have a nice interpretation that relates to the independence of the failure times in the ideal time scale and the usage paths.

It is convenient to introduce some further notation. Let $X_{i}^{*}, i=1, \ldots, n$ denote a sequence of $n$ independent failure times. Under right-censorship, we observe $X_{i}=\min \left(X_{i}^{*}, C_{i}\right)$ and $\delta_{i}=I\left[X_{i}^{*} \leq C_{i}\right], i=1, \ldots, n$, where $C_{i}$ is the censoring time for the $i$ th item and $I[\cdot]$ is the indicator function. Finally, we assume that given $\mathcal{Z}_{i}, X_{i}^{*}$ and $C_{i}$ are independent, $i=1, \ldots, n$.

Under model (1.5), the $\log$-likelihood for $\boldsymbol{\eta}$ is given by

$$
\begin{gathered}
l(\boldsymbol{\eta})=\sum_{i=1}^{n} \int_{0}^{\infty}\left\{\left(\ln \lambda\left[t_{\mathcal{Z}_{i}}(x ; \boldsymbol{\eta})\right]+\ln t_{\mathcal{Z}_{i}}^{\prime}(x ; \boldsymbol{\eta})\right) d N_{i}(x)\right. \\
\left.-Y_{i}(x) \lambda\left[t_{\mathcal{Z}_{i}}(x ; \boldsymbol{\eta})\right] t_{\mathcal{Z}_{i}}^{\prime}(x ; \boldsymbol{\eta}) d x\right\}
\end{gathered}
$$

where $\lambda[u]$ is the hazard function corresponding to $G(u), N_{i}(u)$ is a counting process taking value 0 if $u<X_{i}^{*}$ and 1 if $u \geq X_{i}^{*}, Y_{i}(u)$ is 1 if individual $i$ is at risk of failing at real time $u, 0$ otherwise, and $t_{\mathcal{Z}_{i}}^{\prime}(x ; \boldsymbol{\eta})=d t_{\mathcal{Z}_{i}}(x ; \boldsymbol{\eta}) / d x$. Assuming that the usual regularity conditions hold, we can take the derivative with respect to $\boldsymbol{\eta}$ under the integral sign and obtain the following score function:

$$
\begin{aligned}
U(\boldsymbol{\eta})=\sum_{i=1}^{n} \int_{0}^{\infty} & \left\{\left(\frac{\lambda^{\prime}\left[t_{\mathcal{Z}_{i}}(x ; \boldsymbol{\eta})\right]}{\lambda\left[t_{\mathcal{Z}_{i}}(x ; \boldsymbol{\eta})\right]} \frac{\partial t_{\mathcal{Z}_{i}}(x ; \boldsymbol{\eta})}{\partial \boldsymbol{\eta}}+\frac{\partial t_{\mathcal{Z}_{i}}^{\prime}(x ; \boldsymbol{\eta}) / \partial \boldsymbol{\eta}}{t_{\mathcal{Z}_{i}}^{\prime}(x ; \boldsymbol{\eta})}\right) d N_{i}(x)\right. \\
-Y_{i}(x)\left(\lambda^{\prime}\left(t_{\mathcal{Z}_{i}}(x ; \boldsymbol{\eta})\right] \frac{\partial t_{\mathcal{Z}_{i}}(x ; \boldsymbol{\eta})}{\partial \boldsymbol{\eta}} t_{\mathcal{Z}_{i}}^{\prime}(x ; \boldsymbol{\eta})\right. & \\
+ & \left.\left.\lambda\left[t_{\mathcal{Z}_{i}}(x ; \boldsymbol{\eta})\right] \frac{\partial t_{\mathcal{Z}_{i}}^{\prime}(x ; \boldsymbol{\eta})}{\partial \boldsymbol{\eta}}\right) d x\right\}
\end{aligned}
$$

Following Lin and Ying (1995), let us now replace the unknown cumulative hazard function by its Nelson-Aalen estimator (in scale $t_{\mathcal{Z}}(x ; \boldsymbol{\eta})$ ) and the derivative of the unknown hazard function by zero. After rearranging the terms, we get

$$
\widetilde{U}(\boldsymbol{\eta})=\left.\sum_{i=1}^{n} \int_{0}^{\infty} \frac{\partial \ln t_{\mathcal{Z}_{i}}^{\prime}(x ; \boldsymbol{\eta})}{\partial \boldsymbol{\eta}}\right|_{x=x^{-1}\left[t, \boldsymbol{\eta}, \mathcal{Z}_{i}\right]}\left(d \widetilde{N}_{i}(t ; \boldsymbol{\eta})-\widetilde{Y}_{i}(t ; \boldsymbol{\eta}) d \widetilde{\Lambda}[t ; \boldsymbol{\eta}]\right),
$$


where $x^{-1}\left[t, \boldsymbol{\eta}, \mathcal{Z}_{i}\right]$ is the value of $x$ such that $t_{\mathcal{Z}_{i}}(x ; \boldsymbol{\eta})=t$, and where

$$
\begin{aligned}
\tilde{N}_{i}(t ; \boldsymbol{\eta}) & = \begin{cases}0, & \text { if } t<t_{\mathcal{Z}_{i}}\left(X_{i} ; \boldsymbol{\eta}\right) \\
1, & \text { if } t \geq t_{\mathcal{Z}_{i}}\left(X_{i} ; \boldsymbol{\eta}\right)\end{cases} \\
\tilde{Y}_{i}(t ; \boldsymbol{\eta}) & = \begin{cases}1, & \text { if individual } i \text { is at risk of failing at time } t \text { in scale } t_{\mathcal{Z}}(x ; \boldsymbol{\eta}) \\
0, & \text { otherwise }\end{cases} \\
\tilde{\Lambda}[t ; \boldsymbol{\eta}] & =\text { value of Nelson-Aalen estimator in scale } t_{\mathcal{Z}}(x ; \boldsymbol{\eta}) \text { at time } t .
\end{aligned}
$$

Notice that the score (1.8) is of the form

$$
\sum_{i=1}^{n} \delta_{i}\left(Q_{i}-\overline{Q_{i}}\right)
$$

where $Q_{i}=\partial \ln t_{\mathcal{Z}_{i}}^{\prime}(x ; \boldsymbol{\eta}) / \partial \boldsymbol{\eta}$ and $\overline{Q_{i}}$ is the average of the $Q_{i}$ 's still at risk when item $i$ fails (in scale $t_{\mathcal{Z}}(x ; \boldsymbol{\eta})$ ). This means that score $(1.8)$ is an unbiased estimating function. However this score is not continuous, so we define the estimator of $\boldsymbol{\eta}$ to be $\arg \min _{\boldsymbol{\eta}} \widetilde{U}(\boldsymbol{\eta})^{t} \widetilde{U}(\boldsymbol{\eta})$, the minimum being taken over all $\boldsymbol{\eta}$ in some compact region [Lin and Ying (1995)].

Asymptotic properties of estimators of this type are discussed in Robins and Tsiatis (1992), Ying (1993), Lin and Ying (1995), Bagdonavičius and Nikulin (1997b) and Bordes (1999). In paticular, we have that a relatively accurate approximate $(1-\alpha)$ confidence region for $\boldsymbol{\eta}$ is given by

$$
\left\{\boldsymbol{\eta}: \widetilde{U}(\boldsymbol{\eta})^{t} V^{-1}(\boldsymbol{\eta}) \widetilde{U}(\boldsymbol{\eta}) \leq \chi_{k ; 1-\alpha}^{2}\right\},
$$

where $k=\operatorname{dim}(\boldsymbol{\eta}), \chi_{k ; 1-\alpha}^{2}$ is the $1-\alpha$ quantile of a chi-square distribution on $k$ degrees of freedom and

$$
\begin{aligned}
V(\boldsymbol{\eta})= & \sum_{i=1}^{n} \int_{0}^{\infty}\left[\frac{\sum_{j=1}^{n} \tilde{Y}_{j}(t ; \boldsymbol{\eta}) Q_{j}^{\otimes 2}(t ; \boldsymbol{\eta})}{\sum_{j=1}^{n} \tilde{Y}_{j}(t ; \boldsymbol{\eta})}\right. \\
& \left.-\left(\frac{\sum_{j=1}^{n} \tilde{Y}_{j}(t ; \boldsymbol{\eta}) Q_{j}(t ; \boldsymbol{\eta})}{\sum_{j=1}^{n} \tilde{Y}_{j}(t ; \boldsymbol{\eta})}\right)^{\otimes 2}\right] d \widetilde{N}_{i}(t ; \boldsymbol{\eta}),
\end{aligned}
$$

where

$$
Q_{j}(t ; \boldsymbol{\eta})=\left.\frac{\partial \ln t_{\mathcal{Z}_{j}}^{\prime}(x ; \boldsymbol{\eta})}{\partial \boldsymbol{\eta}}\right|_{x=x^{-1}\left[t, \boldsymbol{\eta}, \mathcal{Z}_{j}\right]}
$$

and, for some vector $u, u^{\otimes 2}$ denotes the outer product $u u^{t}$.

Duchesne (1999) argued that two conditions were necessary for precise estimation of $\boldsymbol{\eta}$ with this and any inference method: large variability in the observed usage paths $\mathcal{Z}_{i}$, and a distribution $\operatorname{Pr}[X>x \mid \mathcal{Z}]$ with small variation. Through simulations, he observed that when these conditions were not met, all 
the estimators considered were biased and inaccurate. For most of the models he considered, the rank-based estimator seemed to be more efficient than the minimum coefficient of variation estimator.

Now, as an illustration, let $z(x)=\theta x$ and put $t_{\mathcal{Z}}(x ; \eta)=(1-\eta) x+\eta \theta x$, i.e. usage is acccumulated at a constant rate, $\theta$, and the ITS is a linear combination of real time and usage. Assuming no censoring and substituting those values into (1.8), we obtain

$$
\widetilde{U}(\eta)=\sum_{i=1}^{n} \frac{\theta_{(i)}-1}{1-\eta+\eta \theta_{(i)}}\left(1-\frac{1}{n}-\frac{1}{n-1}-\cdots-\frac{1}{n-i+1}\right),
$$

where $(i)$ is the label of the $i$ th item to fail in scale $(1-\eta) x+\eta \theta x$. Notice that this last score is the one we would get if we were to test no association between the failure times in scale $(1-\eta) x+\eta \theta x$ and the "covariates" $(\theta-1) /(1-\eta+\eta \theta)$ using rank regression with exponential scores. As $\theta$ completely defines the usage path in this case, this is consistent with the definition of an ITS which implies that the failure times in the ITS are independent of the usage histories.

\subsection{Semiparametric model assessment}

Supposing that a collapsible model is appropriate is a strong assumption. In this section, we propose a simple graphical method based on generalized residuals to assess the adequacy of a specified time scale. We use data provided by Kordonsky and Gertsbakh (1995a) to illustrate the method.

As was mentioned earlier, the failure times in the ITS are independent of the usage histories. This means that if we could plot these times against some features of the usage paths, we should observe only scatter, without any type of trend. Unfortunately, unless the value of $\boldsymbol{\eta}$ is known, we do not observe the failure times in the ITS. Nevertheless, if we have a good estimate of $\boldsymbol{\eta}$, say $\hat{\boldsymbol{\eta}}$, the generalized residuals $t_{\mathcal{Z}_{i}}\left(x_{i} ; \hat{\boldsymbol{\eta}}\right), i=1,2, \ldots, n$ will be close to the failure times in the ITS. We can thus plot these generalized residuals against some features of the usage histories; if we observe any type of trend in any of the plots, this is evidence that $t_{\mathcal{Z}}(x ; \boldsymbol{\eta})$ is not an ITS.

To illustrate this method, let us fit two different time scales to the data provided by Kordonsky and Gertsbakh (1995a). First, let us fit a linear scale of the form $t_{\mathcal{Z}}(x ; \eta)=(1-\eta) x+\eta z(x)$, where $x$ is the number of low stress cycles and $z(x)=\theta x$ is the number of high stress cycles. Using the rank-based estimator of Section 1.3, we obtain $\hat{\eta}=0.868$, which is very close to 0.871 , the value obtained with the minimum coefficient of variation method. The $95 \%$ confidence interval derived from equation $(1.9)$ is $(0.844,0.910)$. Now, let us fit a multiplicative scale of the form $t_{\mathcal{Z}}(x ; \eta)=x^{\eta} z(x)^{1-\eta}$. The estimates of $\eta$ obtained with the rank-based and minimum coefficient of variation methods 
are respectively 0.800 and 0.804 . The $95 \%$ confidence interval for $\eta$ from (1.9) is $(0.662,0.930)$. Figure 1 shows a plot of the generalized residuals against the path parameter $\theta$ for both scales. There does not seem to be any apparent trend in the plot for the linear scale, but there is a definite quadratic trend in the plot for the multiplicative scale. The linear scale seems to be more appropriate in this case.
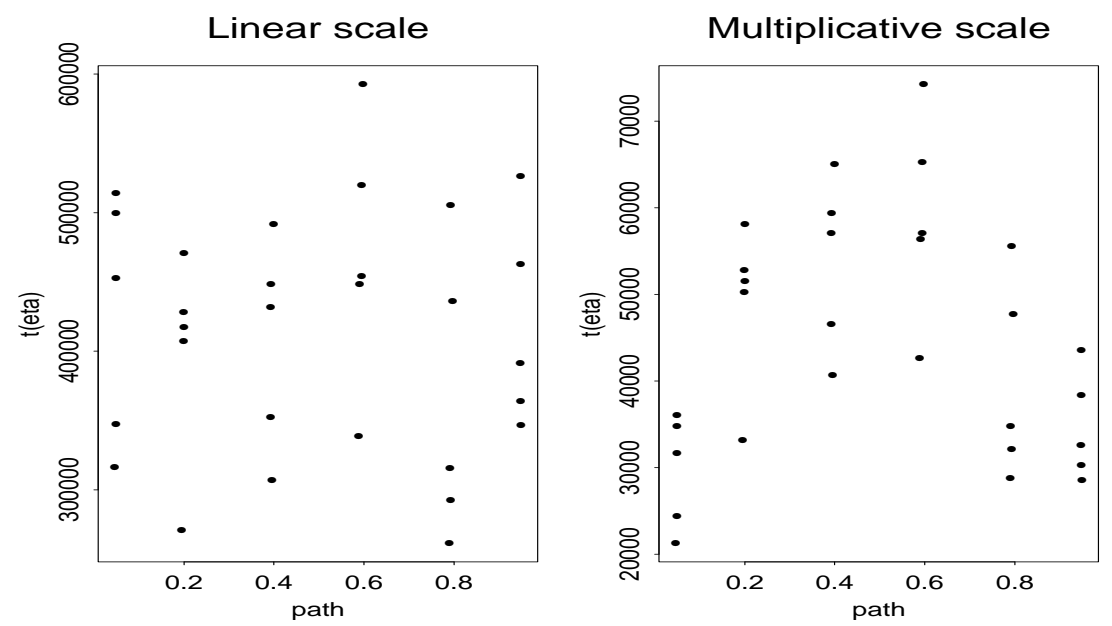

Figure 1. Generalized residual plots: failure times in scales $0.132 x+0.868 z(x)$ (left) and $x^{0.200} z(x)^{0.800}$ (right) versus the proportion of high stress cycles.

\subsection{Nonparametric age curves}

The nice interpretation of a model collapsible in $x, z_{1}(x), \ldots, z_{p}(x)$, is that the items travel on a path that crosses age curves. For example if a model is collapsible in $x$ and $z(x)$, and if a linear scale of the form $0.2 x+0.8 z(x)$ is ideal, then all the points in the positive quadrant on a line parallel to the line $0.2 x+0.8 z=c$ have the same age in the ITS, i.e. $\operatorname{Pr}[X>x \mid \mathcal{Z}]$ is constant over these lines. An interesting consequence of this is that if we could draw the level curves of $\operatorname{Pr}[X>x \mid \mathcal{Z}]$ nonparametrically, we would have a picture of the form of the ITS function $\Phi\left[x, z_{1}(x), \ldots, z_{p}(x)\right]$.

Little work has been done in this area. In the case where the usage paths can be completely described by a finite vector of parameters, say $\boldsymbol{\theta}$, we can try to adapt some of the nonparametric quantile regression algorithms described in the literature [Lejeune and Sarda (1988), Cole and Green (1992)]. However for the simple linear and multiplicative scales described above, these nonparametric 
quantile regression methods exhibit some serious edge effects that distort the picture of the ideal time scale function.

We can adopt another approach to nonparametric estimation of the ideal time scale when the usage paths are such that we have several observations distributed among a few groups of similar paths. Then we can group those similar paths together, estimate a given quantile of $X$ in each group of paths, then draw segments between the estimated quantiles. These segments should form a curve that will be representative of the form of the ideal time scale. To illustrate, consider the following two models: $\operatorname{Pr}\left[X_{i}>x \mid \mathcal{Z}_{i}\right]=G\left(x+z_{i}(x)\right)$ and $\operatorname{Pr}\left[X_{i}>x \mid \mathcal{Z}_{i}\right]=G\left(x^{2}+z_{i}(x)^{2}\right)$, and assume that $z_{i}(x)=\theta_{i} x$, where the $\theta_{i}$ 's vary in $[0, \infty)$ within the population of items. We generated a sample of size 400 from both models with $G$ being the Weibull(shape=5, scale=9) and $\operatorname{atan}(\Theta) \sim$ Uniform $(0, \pi / 2)$. Then for both samples, we split the positive quadrant in 4 slices, estimated the quintiles of $X$ in each slice, then drew lines that linked corresponding quintiles together. The results are shown in Figure 2 .

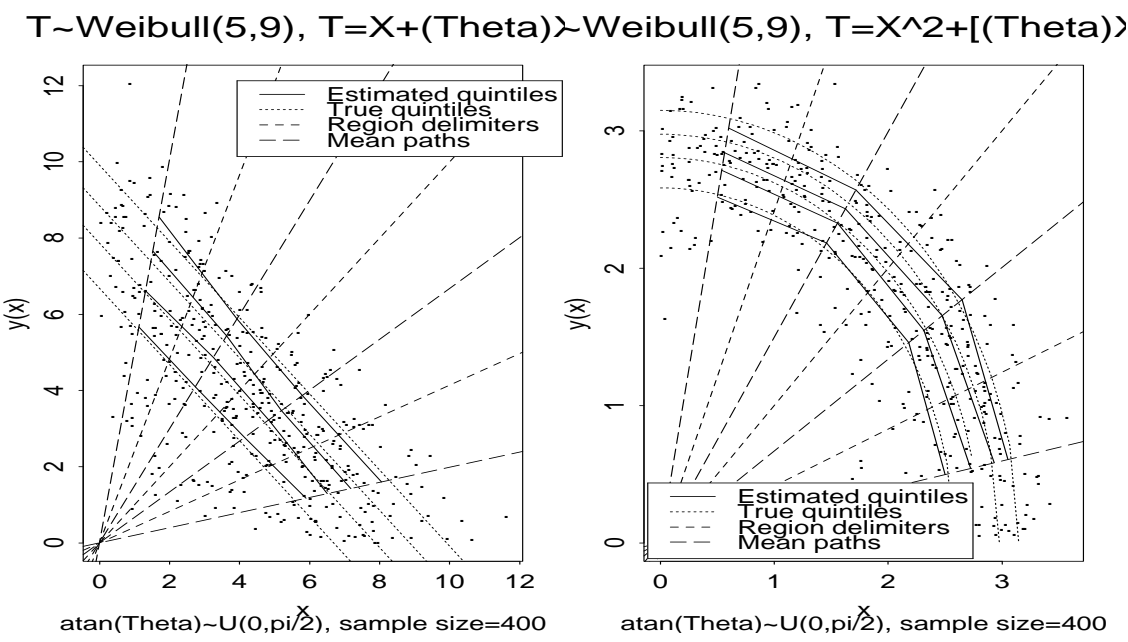

Figure 2. Age curves drawn nonparametrically.

The two plots in Figure 2 clearly identify the linear and quadratic forms of the ideal time scales. However, these two samples were "nicer" than the samples we are likely to encounter in practice; we had a large number of observations and the usage paths were nicely spread over the whole positive quadrant. Fine tuning this age curve drawing method is required. Perhaps introducing smoothing or combining this method with a quantile regression algorithm could yield good nonparametric estimates of the ITS. 


\subsection{Conclusion}

The selection of an ideal collapsible time scale is not a trivial exercise. When a parametric form for the time scale has been selected, semiparametric methods based on ranks allow for precise parameter estimation, even under rightcensoring, provided that enough variability in the covariate histories is observed and that these covariates are good predictors of failure.

Supposing that a simple time scale is ideal is a strong assumption that should be verified. A simple graphical method based on semiparametric generalized residuals has been proposed. More formal semiparametric methods would be desirable. Nonparametric estimation of the ideal time scale has not yet been investigated thoroughly, but it seems to be possible in the case of collapsible models.

\section{Ackowledgements}

I would like to thank the Natural Sciences and Engineering Research Council of Canada for their financial support and professor Jerry Lawless for his helpful advise and comments.

\section{References}

1. Bagdonavičius, V.B. and Nikulin, M.S. (1997a). Transfer functionals and semiparametric regression models, Biometrika, 84, 365-378.

2. Bagdonavičius, V.B. and Nikulin, M.S. (1997b). Asymptotical analysis of semiparametric models in survival analysis and accelerated life testing, Statistics, 29, 261-283.

3. Bordes, L. (1999). Semiparametric additive accelerated life models, Scandinavian Journal of Statistics, 26, pp. 345-361.

4. Cole, T.J. and Green, P.J. (1992). Smoothing reference centile curves: the lms method and penalized likelihood. Statistics in Medicine, 11, 13051319 .

5. Cinlar, E. and Ozekici, S. (1987). Reliability of complex devices in random environments, Probability in the Engineering and Informational Sciences, 1, $97-115$.

6. Duchesne, T. (1999). Multiple Time Scales in Survival Analysis, Doctoral dissertation, University of Waterloo. 
7. Duchesne, T. and Lawless, J. (2000). Alternative time scales and failure time models, Lifetime Data Analysis, to appear.

8. Farewell, V.T. and Cox, D.R. (1979). A note on multiple time scales in life testing, Applied Statistics, 28, 73-75.

9. Kordonsky, K.B. and Gertsbakh, I. (1993). Choice of the best time scale for system reliability analysis, European Journal of Operational Research, 65, 235-246.

10. Kordonsky, K.B. and Gertsbakh, I. (1995a). System state monitoring and lifetime scales-I, Reliability Engineering and System Safety, 47, 1-14.

11. Kordonsky, K.B. and Gertsbakh, I. (1995b). System state monitoring and lifetime scales-II, Reliability Engineering and System Safety, 49, 145-154.

12. Kordonsky, K.B. and Gertsbakh, I. (1997). Multiple time scales and the lifetime coefficient of variation: Engineering applications, Lifetime Data Analysis, 2, 139-156.

13. Lawless, J.F., Hu, J. and Cao, J. (1995). Methods for the estimation of failure distributions and rates from automobile warranty data, Lifetime Data Analysis, 1, 227-240.

14. Lejeune, M.G. and Sarda, P. (1988). Quantile regression: a nonparametric approach, Computational Statistics and Data Analysis, 6, 229-239.

15. Lin, D.Y. and Ying, Z. (1995). Semiparametric inference for the accelerated life model with time-dependent covariates, Statistical Planning and Inference, 44, 47-63.

16. Oakes, D. (1995). Multiple time scales in survival analysis, Lifetime Data Analysis, 1, 139-156.

17. Robins, J. and Tsiatis, A.A. (1992). Semiparametric estimation of an accelerated failure time model with time-dependent covariate, Biometrika, 79, 311-319.

18. Ying, Z. (1993). A large sample study of rank estimation for censored regression data, Annals of Statistics, 21, pp. 76-99. 